\title{
Anatomical study of anterior supramalleolar artery and its potential application to design a bi-foliate fasciocutaneous flap
}

\author{
Ji-Yin He ${ }^{1}$, Shih-Heng Chen ${ }^{2}$, Kannan Karuppiah Kumar ${ }^{3}$, Zhi-Hong Fan ${ }^{1}$, Jie Lao ${ }^{4}$, Huey Tien ${ }^{3}$ \\ Department of Plastic Surgery, School of Medicine, Renji Hospital, Shanghai Jiaotong University, Shanghai, China ${ }^{1}$, \\ Department of Plastic and Reconstructive Surgery, College of Medicine, Chang Gung Memorial Hospital, Chang Gung \\ University, Taoyuan, Taiwan², Christine M. Kleinert Institute for Hand and Microsurgery, Louisville, Kentucky, USA ${ }^{3}$, \\ Department of Hand Surgery, Huashan Hospital Affiliated Fudan University, Shanghai, China ${ }^{4}$
}

Address for correspondence: Dr. Huey Tien, Christine M. Kleinert Institute for Hand and Microsurgery, 225 Abraham Flexner Way, Louisville, Kentucky 40202, USA. E-mail: htien@kleinertkutz.com

\section{ABSTRACT}

Purpose: A further understanding of the anterior supramalleolar artery (ASMA) and its potential applications in reconstructive surgery. Materials and Methods: A total of 24 fresh lower limbs from fresh cadavers were injected with red latex for dissection. The type of origin, course, diameter of the pedicle, and the distance between the origin of the ASMA from the anterior tibial artery to the extensor retinaculum (O-R) were recorded. Bi-foliate fasciocutaneous flaps were harvested using the branches of the ASMA. Results: We found four types of origin of the ASMA, and we have accordingly classified them into four types. 10 of them were type $A, 7$ were type $B, 6$ were type $C$ and 1 was type $D$. The mean O-R (origin of ASMA to retinaculum) distance was $2.0 \pm 0.8 \mathrm{~cm}$. The diameter of the medial branch (D1), the diameter of the lateral branch (D2), and the diameter of artery stem (D3) (only in type A) were $1.0 \pm 0.2 \mathrm{~mm}, 0.8 \pm 0.3 \mathrm{~mm}, 1.1 \pm 0.2 \mathrm{~mm}$, respectively. The mean pedicle length of the lateral flap (L1) and medial flap (L2) were $5.1 \pm 1.0 \mathrm{~cm}$ and $3.7 \pm 0.6$ $\mathrm{cm}$, respectively. Conclusions: The ASMA exists constantly with four different types of origin. Its sizable diameter and lengthy pedicle make it suitable for bi-foliate fasciocutaneous flap transfer.

\section{KEY WORDS}

Anterior supramalleolar artery; bipaddle flaps; fasciocutaneous flaps; multiple defects

\section{INTRODUCTION}

he three main arteries of the leg, the anterior tibial artery (ATA), posterior tibial artery and peroneal artery, form a dense vascular network around the

\begin{tabular}{|l|l|}
\hline \multicolumn{2}{|c|}{ Access this article online } \\
\hline Quick Response Code: & Website: \\
\hline & www.ijps.org \\
\cline { 2 - 2 } & Dol: \\
\hline
\end{tabular}

distal leg, ankle, and foot, ${ }^{[1-4]}$ which ramifies perforators as the basis of all kinds of pedicle flaps such as lateral or medial malleolar perforator flaps. There is plenty of literature describing the anatomy and clinical application of perforators of the peroneal artery ${ }^{[5-9]}$ and posterior tibial artery ${ }^{[10-13]}$ around the distal lower leg. However, literature regarding perforator flaps of ATA or anterior supramalleolar artery (ASMA), one of the major branches of distal ATA, are scant. ${ }^{[14-17]}$ These papers either mention the existence of the perforators from ATA or ASMA, or describe their communication with the medial and lateral supramalleolar arteries. None of them have a detailed anatomical study on ASMA.

Indian Journal of Plastic Surgery January-April 2015 Vol 48 Issue 1 
The objective of this study was to identify the anatomic parameters of the ASMA and its perforators that would enable microsurgeons to harvest potentially multi-paddle flaps or composite flaps from the foot and ankle region. We try to preserve the ATA intact after we harvest the composite flaps around the ankle using ASMA. We also want to use the ATA as the only one pedicle and harvest multi-paddle flaps, decreasing the microsurgical risk greatly and minimizing the sacrifice of the blood supply to the ankle and foot.

\section{MATERIALS AND METHODS}

After the study was approved by the University of Louisville IRB; 24 lower extremities of fresh human cadavers were studied. The ATA was cannulated and injected with $20 \mathrm{ml}$ of red latex (Microfilm, FLOW TECH, Inc.,) at the mid-point of lower leg where ATA can be identified between the extensor digitorum longus and tibialis anterior muscles. After injection, it took 24 hours for the latex to solidify. Then, careful dissection along the distal $1 / 3$ of the lower leg, the ankle, and the proximal foot $(8-10 \mathrm{~cm}$ above the lateral malleolus and $1-2 \mathrm{~cm}$ anterior to the edge of the fibula) was performed to observe the origin and the course of the ASMA. The distance between the origin of ASMA and the extensor retinaculum $(\mathrm{O}-\mathrm{R})$ was recorded. The diameters of the ASMA proper as well as its branches (A set of fixed-width hooks was used for measurement), and the length of ASMA were measured. The collaterals between ASMA and the medial and lateral supramalleolar arteries and the number of perforators were observed. Different views of photographs were taken during the dissection. Chi-square test was used for this study to analyse the variation of the O-R. Fasciocutaneous flaps with multiple skin paddles were elevated based on the perforators to demonstrate its potential application.

\section{RESULTS}

In all the 24 specimens, the ASMA could be identified during dissection. We classified the ASMA into four types according to the pattern of origin from the ATA [Figures 1-4]. Type A $(n=10)$ ASMA originates from the ATA with a $1-1.5 \mathrm{~cm}$ long main trunk and then gives off its lateral and medial branches [Figure 1a and b]; each consists of 2-3 perforators. In type B $(n=7)$, the ASMA does not have a main trunk, and its lateral and medial branches stem directly from ATA with the sites of origin at the same level [Figure 2a and b]. Type $C(n=6)$ is similar to type $\mathrm{B}$, with the lateral and medial branches arising from the ATA but at different origins [Figure 3a and b]. Type $\mathrm{D}(n=1)$ has 2 medial, and 1 lateral branch arising from the ATA [Figure $4 a$ and $b$ ].

The mean distance between the origin of the proximal branch of ASMA and the superior margin of the extensor retinaculum $(\mathrm{O}-\mathrm{R})$ was $2.0 \pm 0.8 \mathrm{~cm}$. The mean diameters of the medial branch (D1), lateral branch (D2)

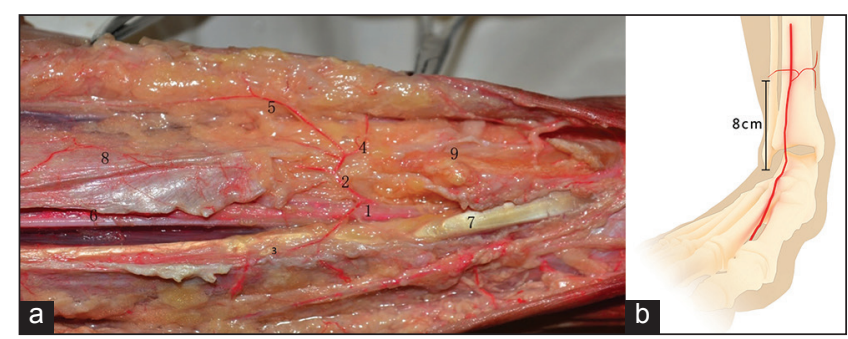

Figure 1: (a) Type A - the anterior supramalleolar artery originates as an artery stem. (1) Short artery stem; (2) lateral branches; (3) medial branches; (4) descendent perforators; (5) ascendant perforators. (6) Proximal end of anterior Tibial artery (ATA); (7) distal end of ATA; (8) blood supply to Tibia; (9) extensor retinaculum (b) the anterior supramalleolar artery originates as a $1-1.5 \mathrm{~cm}$ short artery stem and then ramifies lateral and medial branches. The medial branch gives off ascendant perforators and descendent perforators

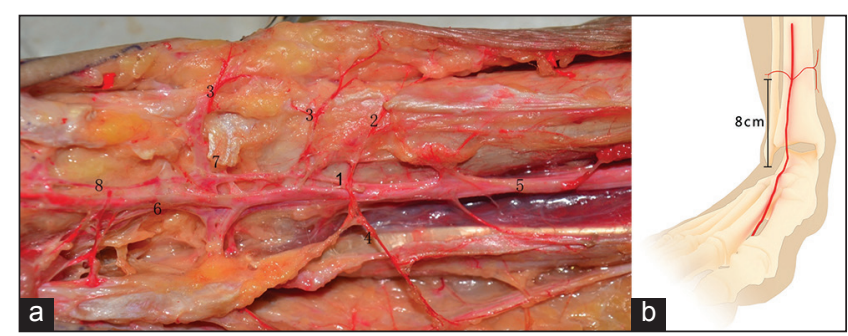

Figure 2: (a) Type B - the anterior supramalleolar artery originates as lateral and medial artery branches at the same point. (1) Medial branches; (2) ascendant perforators. (3) Descendent perforators; (4) lateral branches; (5) proximal end of anterior tibial artery (ATA); (6) distal end of ATA; (7) extensor retinaculum; (8) blood supply to tibia (b) the anterior supramalleolar artery orients as lateral and medial artery branches at the same point. The medial branch gives off ascendant perforators and descendent perforators

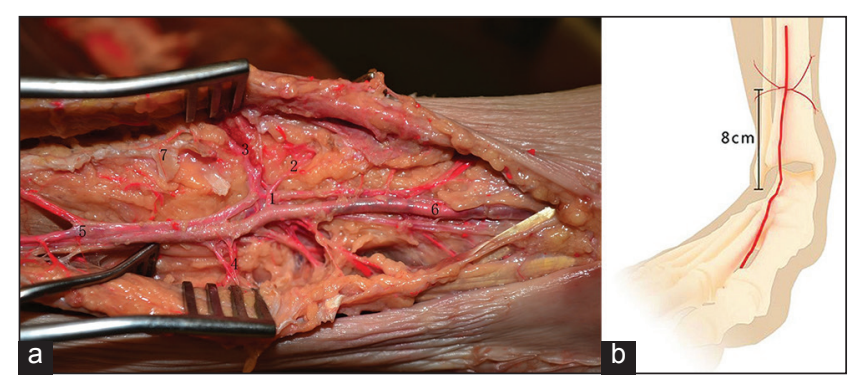

Figure 3: (a) Type $\mathrm{C}-$ the anterior supramalleolar artery originates as lateral and medial artery branches at the different point. (1) Medial branches; (2) ascendant perforators. (3) Descendent perforators; (4) lateral branches; (5) proximal end of anterior tibial artery (ATA); (6) distal end of ATA; (7) extensor retinaculum (b) the anterior supramalleolar artery orients as lateral and medial artery branches at different point. The medial branch gives off ascendant perforators and descendent perforators 


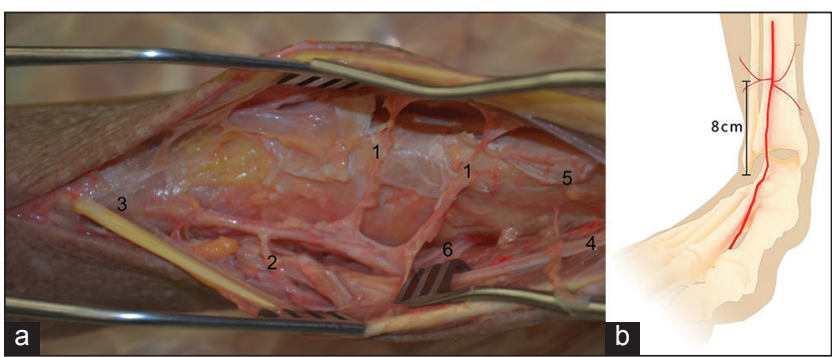

Figure 4: (a) Type D - the anterior supramalleolar artery originates as lateral and two medial artery branches at the different point. (1) Medial branches; (2) lateral branches; (3) proximal end of anterior tibial artery (ATA); (4) distal end of ATA; (5) extensor retinaculum (b) the anterior supramalleolar artery orients as one lateral and two medial arteries branches at different point

and the main trunk of the ASMA (D3) (only in type A) were $1.0 \pm 0.2 \mathrm{~mm}, 0.8 \pm 0.3 \mathrm{~mm}$ and $1.1 \pm 0.2 \mathrm{~mm}$, respectively. The mean feasible length of the pedicles of the lateral flap (L1) and medial flap (L2) were $5.1 \pm 1.0 \mathrm{~cm}$ and $3.7 \pm 0.6 \mathrm{~cm}$, respectively. All data is summarised in Table 1. Bi-foliate fasciocutaneous flaps can be harvested based on the medial and lateral branches of the type $A$ ASMA with preservation of the ATA [Figure 5].

\section{DISCUSSION}

Huber ${ }^{[18]}$ dissected 200 feet and was the first to describe the ASMA as a vessel arising about $5 \mathrm{~cm}$ above the ankle joint. Satoh et al. and Wee $e^{[14,15]}$ described that this septocutaneous branch of ATA runs through the anterior crural septum between the tendons of tibialis anterior and extensor hallucis longus and gives out 3 perforators. Satoh et al. ${ }^{[14]}$ further named the reverse-flow flap based on the perforators of this artery as anterior tibial type III. Later research ${ }^{[16]}$ showed that ASMA is always found in the distal $1 / 3$ of the lower leg and appears $8-10 \mathrm{~cm}$ above the lateral malleolus, $1-2 \mathrm{~cm}$ anterior to the edge of the fibula. In 2003, Koshima ${ }^{[19]}$ dissected 4 ankles and described that the ASMA has 1-2 perforators just above the extensor retinaculum and gives off the anterolateral and anteromedial branches. These studies recognised the existence of ASMA and its perforators with possible clinical applications. However, we still lack detailed anatomical knowledge of its origin, calibre and so on.

The diameters of the lateral and medial branches of ASMA are $0.8 \pm 0.3 \mathrm{~mm}$ and $1.0 \pm 0.2 \mathrm{~mm}$, respectively. The feasible pedicle lengths of these two branches are $5.1 \pm 1.0 \mathrm{~cm}$ and $3.7 \pm 0.6 \mathrm{~cm}$, respectively. Thus, an island flap can be harvested based on one or both of the branches for defect coverage around the ankle. In addition, due to its collateral connection with the

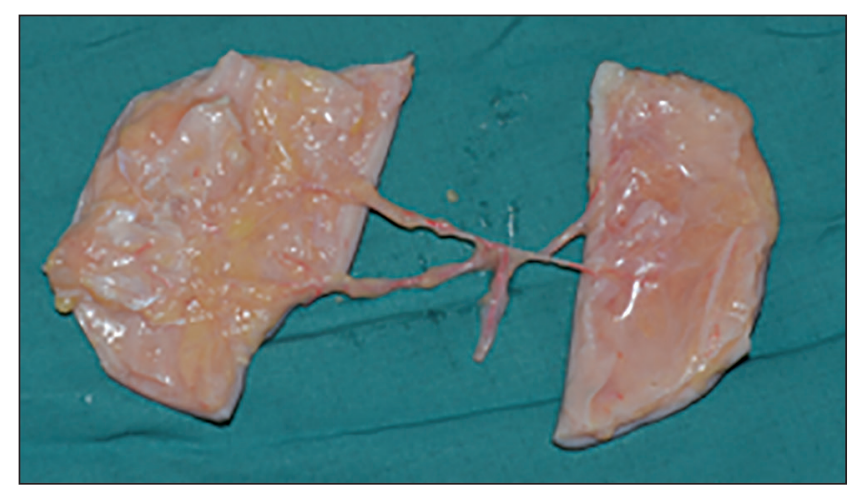

Figure 5: The biofoliate flap using type A anterior supramalleolar artery

Table 1: Data of 24 specimens of the anterior supramalleolar artery

\begin{tabular}{lcccccc}
\hline Type & O-R & D1 & D2 & D3 & L1 & L2 \\
\hline B & 2 & 1 & 0.8 & & 6 & 4.5 \\
A & 2 & 1 & 0.8 & 1.2 & 5.5 & 4 \\
B & 2.5 & 1 & 0.6 & & 6 & 4 \\
A & 1.5 & 1 & 0.8 & 1.2 & 3.5 & 4.5 \\
B & 2.5 & 0.6 & 0.4 & & 3 & 3.5 \\
A & 1.5 & 1 & 0.6 & 1.2 & 4 & 3.5 \\
B & 2 & 1.2 & 1.4 & & 5 & 3 \\
A & -1.5 & 0.6 & 0.4 & 0.8 & 4.5 & 3.5 \\
C & 3 & 1 & 0.8 & & 4.5 & 4 \\
C & 2.5 & 1.2 & 0.8 & & 6 & 3 \\
A & 1 & 0.6 & 1 & 1.2 & 6 & 4 \\
C & 2 & 1.2 & 0.8 & & 5.5 & 4 \\
A & 2.5 & 1.2 & 0.6 & 1.4 & 6 & 3.5 \\
A & 1.5 & 0.8 & 0.6 & 1 & 6 & 4.5 \\
B & 2 & 0.8 & 0.4 & & 5.5 & 3.5 \\
C & 3 & 0.8 & 1 & & 6 & 5 \\
B & 2.5 & 1.2 & 0.8 & & 4 & 3 \\
C & 3.5 & 0.8 & 1.4 & & 6 & 4 \\
B & -1 & 1 & 0.8 & & 5 & 3.5 \\
A & -1 & 1 & 0.8 & 1.2 & 5.5 & 3 \\
A & 0 & 1 & 0.8 & 1.2 & 5 & 3 \\
A & 2 & 0.8 & 0.6 & 1 & 4 & 3 \\
C & 3 & 1 & 0.8 & & 7 & 3.5 \\
D & -1 & 1 & 0.8 & & 4 & 3 \\
\hline Typ & 1 & 1yp & 0.5 & & & \\
\hline
\end{tabular}

Type: Different types of the origin of the anterior supramalleolar artery; O-R: The distance between the origin of the proximal branch of the anterior supramalleolar artery to superior margin of the extensor retinaculum $(\mathrm{cm})$; D1: The diameter of the medial branch $(\mathrm{mm})$; D2: The diameter of the lateral branch $(\mathrm{mm})$; D3: The diameter of artery stem (Type A) (mm); L1: The possible pedicle length of the lateral flap $(\mathrm{cm})$; L2: The possible pedicle length of medial flap $(\mathrm{cm})$

posterior tibial artery and peroneal artery, a reverseflow anterolateral or anteromedial flap can also be designed as described in literature. ${ }^{[5-13]}$ The flaps with ASMA are very useful especially for patients who suffer from refractory malleolar ulcers due to paralysis, diabetes mellitus, venous stasis, or vasculitis. For patients who have cosmetic consideration, perforatorbased adipofascial flap is also a good option with the use of ASMA. ${ }^{[20-24]}$

Indian Journal of Plastic Surgery January-April 2015 Vol 48 Issue 1 
The perforators of the ASMA are suitable for a bi-foliate design in situations such as simultaneous defects of the volar and dorsal aspects of the hand. The flap based on ASMA is pliable and thin, which makes it an excellent option for hand and wrist coverage. If there is a simultaneous metacarpal deficiency at the same time, which can present especially in high-energy injuries, vascularised bone graft can also be harvested easily supplied by 1-2 perforators according to research by Gilbert et al. ${ }^{[25]}$ An additional advantage of this perforator flap is that the ATA can be preserved in type A ASMA, and morbidity of the donor site can be minimised. Based on different types of ASMA, its lumen size can vary from $0.8 \pm 0.3$ to $1.1 \pm 0.2 \mathrm{~mm}$, which is still suitable for microvascular anastomosis.

A difficult problem that a hand surgeon encounters is the need for multiple flaps to cover soft tissue loss in multiple digits. Conventional methods include inserting the hand into an abdominal pocket for several weeks followed by skin graft, using a large flap to cover all the defects together, or covering the wounds with several free flaps at the same time. Often, these patients will still exhibit stiff digits and a bulky hand. And combined multiple single flaps would increase the surgical risk exponentially. In this instance, we propose a novel idea of using a multiple-foliate flap based on different perforators of the lateral and medial branches of the ASMA to cover multiple digits at the same time. These branches all derive from the same trunk-The ATA and its terminal branch. This design provides the capability of harvesting up to 2-3 skin paddles based on one sizable pedicle, the ATA. It also facilitates microvascular anastomosis easily, which decreases the risk of flap failure. The terminal branches of the peroneal artery to ankle and foot, as well as the branches of posterior tibial artery to ankle and foot are also preserved. In this way, the vascular network of the ankle and foot is still preserved, and donor site morbidity is significantly decreased.

There are several factors which should be kept in mind during the clinical application of ASMA based flaps. When the skin is opened, the lateral and medial branches of the ASMA are generally visible. If we want only the stem artery and their branches, we can just follow the course of these branches and easily find the origin of these branches. A minimal necessary incision is enough. Then, we can harvest these flaps safely in a minimally invasive fashion. However, in any situation it would be more effective to repair the extensor retinaculum carefully and immobilize the foot for 3-4 weeks.

Further study and long-term follow-up are needed to understand the blood supply of the ankle and foot after harvesting the ASMA-based flap.

\section{CONCLUSIONS}

The anatomy of ASMA is fairly constant and enables the harvest of reliable flaps. It can be classified into four different types. Its sizable diameter and lengthy pedicle make it suitable as bi-foliate fasciocutaneous flaps or composite flaps.

\section{REFERENCES}

1. Ballmer FT, Hertel R, Noetzli HP, Masquelet AC. The medial malleolar network: A constant vascular base of the distally based saphenous neurocutaneous island flap. Surg Radiol Anat 1999;21:297-303.

2. Ozdemir R, Kocer U, Sahin B, Oruc M, Kilinc H, Tekdemir I. Examination of the skin perforators of the posterior tibial artery on the leg and the ankle region and their clinical use. Plast Reconstr Surg 2006;117:1619-30.

3. Ozalp T, Masquelet AC, Begue TC. Septocutaneous perforators of the peroneal artery relative to the fibula: Anatomical basis of the use of pedicled fasciocutaneous flap. Surg Radiol Anat 2006;28:54-8.

4. Vazquez T, Rodríguez-Niedenfuhr M, Parkin I, Viejo F, Sanudo J. Anatomic study of blood supply of the dorsum of the foot and ankle. Arthroscopy 2006;22:287-90.

5. Carriquiry C, Aparecida Costa M, Vasconez LO. An anatomic study of the septocutaneous vessels of the leg. Plast Reconstr Surg 1985;76:354-63.

6. Masquelet AC, Beveridge J, Romana C, Gerber C. The lateral supramalleolar flap. Plast Reconstr Surg 1988;81:74-81.

7. Sham E, Choi WT, Flood SJ. Lateral supramalleolar flap in reconstruction of pressure ulcers in patients with spinal cord injury. ANZ J Surg 2008;78:167-71.

8. Hamdi MF, Khlifi A. Lateral supramalleolar flap for coverage of ankle and foot defects in children. J Foot Ankle Surg 2012;51:106-9.

9. Akita S, Mitsukawa N, Rikihisa N, Himeta J, Kubota Y, Shimada N, et al. Descending branch of the perforating branch of the peroneal artery perforator-based island flap for reconstruction of the lateral malleolus with minimal invasion. Plast Reconstr Surg 2013;132:461-9.

10. Masquelet AC, Romana MC. The medialis pedis flap: A new fasciocutaneous flap. Plast Reconstr Surg 1990;85:765-72.

11. Satoh K, Sakai M, Hiromatsu N, Ohsumi N. Heel and foot reconstruction using reverse-flow posterior tibial flap. Ann Plast Surg 1990;24:318-27.

12. Koshima I, Soeda S. Free posterior tibial perforator-based flaps. Ann Plast Surg 1991;26:284-8.

13. Koshima I, Moriguchi T, Ohta S, Hamanaka T, Inoue T, Ikeda A. The vasculature and clinical application of the posterior tibial perforator-based flap. Plast Reconstr Surg 1992;90:643-9.

14. Satoh K, Yoshikawa A, Hayashi M. Reverse-flow anterior tibial flap type III. Br J Plast Surg 1988;41:624-7. 
15. Wee JT. Reconstruction of the lower leg and foot with the reverse-pedicled anterior tibial flap: Preliminary report of a new fasciocutaneous flap. Br J Plast Surg 1986;39:327-37.

16. Satoh K, Aoyama R, Onizuka T. Comparative study of reverse flow island flaps in the lower extremities - peroneal, anterior tibial, and posterior tibial island flaps in 25 patients. Ann Plast Surg 1993;30:48-56.

17. Koshima I, Yamamoto $\mathrm{H}$, Moriguchi $\mathrm{T}$, Kawada $\mathrm{S}$, Ono $\mathrm{Y}$. Combined anteroposterior tibial perforator-based flap with a vascularized deep peroneal nerve for repair of facial defect. Ann Plast Surg 1994;33:421-5.

18. Huber JF. The arterial network supplying the dorsum of the foot. Anat Rec 1941;80:373-91.

19. Koshima I, Itoh S, Nanba Y, Tsutsui T, Takahashi Y. Medial and lateral malleolar perforator flaps for repair of defects around the ankle. Ann Plast Surg 2003;51:579-83.

20. Le Nen D, Beal D, Person H, Lefevre C, Sénécail B. Anatomical basis of a fascio-cutaneous pedicle flap based on the infero-lateral collateral artery of the leg. Surg Radiol Anat 1994;16:3-8.

21. Touam C, Rostoucher P, Bhatia A, Oberlin C. Comparative study of two series of distally based fasciocutaneous flaps for coverage of the lower one-fourth of the leg, the ankle, and the foot. Plast Reconstr Surg 2001;107:383-92.

22. Lee YH, Rah SK, Choi SJ, Chung MS, Baek GH. Distally based lateral supramalleolar adipofascial flap for reconstruction of the dorsum of the foot and ankle. Plast Reconstr Surg 2004;114:1478-85.

23. Kilinc $\mathrm{H}$, Bilen BT, Arslan A. A novel flap to repair medial and lateral malleolar defects: Anterior tibial artery perforator-based adipofascial flap. Ann Plast Surg 2006;57:396-401.

24. Demirtas Y, Ayhan S, Sariguney Y, Findikcioglu F, Cukurluoglu O, Latifoglu $O$, et al. Distally based lateral and medial leg adipofascial flaps: Need for caution with old, diabetic patients. Plast Reconstr Surg 2006;117:272-6

25. Gilbert BJ, Horst F, Nunley JA. Potential donor rotational bone grafts using vascular territories in the foot and ankle. J Bone Joint Surg Am 2004;86-A:1857-73.

How to cite this article: He J, Chen S, Kumar KK, Fan Z, Lao J, Tien $\mathrm{H}$. Anatomical study of anterior supramalleolar artery and its potential application to design a bi-foliate fasciocutaneous flap. Indian J Plast Surg 2015;48:17-21.

Source of Support: Nil, Conflict of Interest: None declared.

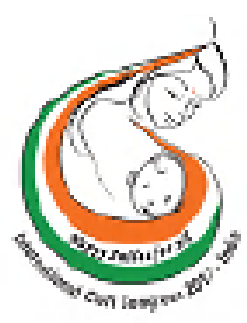

International Congress of the Confederation of Cleft Lip, Palate and Related Craniofacial Anomalies

Dates : 8-11th February 2017

Venue : Mahabalipuram, Chennai, India

Theme: Think, Treat and Teach

webpage : www.cleft2017.org

contact : infoedeft2017.org

Hight Lights:

Event awaited by all the cleft and craniofacial professionals happening every 4 vears

CME for all muti-specialties

Task Force brining out consensus among the professional world across

10 scholarships (each $\$ 2500$ ) for the best papers 10 iunior presentation

Exciting venue beach resort of Mahabalipuram 